\title{
Estimation of the Frequencies of Thin Elastic Plates With Free Edges
}

\author{
Tosio Kato, ${ }^{1}$ Hiroshi Fujita, ${ }^{2}$ Yoshimoto Nakata, ${ }^{2}$ and Morris Newman
}

\begin{abstract}
A variational method is proposed for calculating the frequencies of thin elastic plates with free edges, with rigorous error estimates. As a numerical example, the fundamental frequency of a square plate with the Poisson ratio 0.225 is calculated with a satisfactory result, the possible relative error being less than $1 / 2000$. Generalization to more complicated boundary conditions is straightforward.
\end{abstract}

\section{Introduction}

1.1. The present paper is concerned with the study of the vibration of a thin elastic plate with free edges. Let us consider a plate that occupies in its natural condition a plane domain $D$ in the $x y$-plane bounded by a sufficiently regular contour $C$. It is known $[1,2]^{3}$ that the problem of determining the frequencies of this plate may be reduced to the following eigenvalue problem, denoted by (Pr.), for the differential system consisting of the differential equation

$$
\Delta^{2} w=\left(\frac{\partial^{2}}{\partial x^{2}}+\frac{\partial^{2}}{\partial y^{2}}\right)^{2} w=\lambda w \quad \text { in } D,
$$

with the boundary conditions

$$
\left.\begin{array}{rr}
\frac{\partial \Delta w}{\partial n}+(1-\mu) \frac{d}{d s} \frac{\partial^{2} w}{\partial n \partial t}=0 & \text { on } C, \\
\mu \Delta w+(1-\mu) \frac{\partial^{2} w}{\partial n^{2}}=0 & \text { on } C,
\end{array}\right\}
$$

where $\mu$ is an elastic constant, called Poisson's ratio, such that $0 \leqq \mu<\frac{1}{2}$ (the mathematical theory applies for $0 \leqq \mu<1$ ), and where $s$ is the arc length parameter of $C$ and $\partial / \partial n$, $\partial / \partial t$ represent respectively the derivatives in the directions of the outer normal vector $n$ and the tangential vector $t$ to $C$ at the point under consideration. In the case of angular points on $C$, where the direction of $n$ varies discontinuously with a jump, $w$ is subjected to an additional boundary condition [1]: $\partial^{2} w / \partial n \partial t$ at a point $P$ on $C$ tends to the same value as $P$ approaches any one of the angular points from either side.

The main object is to propose a variational method that enables us to calculate approximate values of the eigenvalues with a rigorous estimate of the error; in other words, to calculate upper and lower bounds for the eigenvalues. Such a method seems interesting and important from the viewpoint of application, inasmuch as it appears, even for most elementary shapes of $C$, almost impossible to obtain an explicit solution of (Pr.). On the other hand, usual approximating methods applied to (Pr.) seem to suffer from the lack of error estimation. For example, the well-known method of Ritz is applicable and was actually applied to the case of a square plate [2]. Although we know empirically that his method provides us with approximate values whose accuracy is satisfactory in most cases dealt with in practice, it should be noted that they are only known to be upper bounds for the quantities in question and nothing can be said about the error bounds. See [10] for a comparative discussion of existing methods, some of which furnish lower bounds.

\footnotetext{
1 University of Tokyo, American University, and National Bureau of Standards.

${ }^{2}$ Department of Physies, University of Tokyo.

${ }^{3}$ Figures in brackets indicate the literature references at the end of this paper.
} 
1.2. Our method is based on the following theorem, proved in [3], concerning a selfadjoint operator in a Hilbert space ${ }^{4}$ of the type $T^{*} T$, which reads, in a form slightly modified for our later convenience,

Theorem $1 .^{5}$ Let $T$ be a closed linear operator with its domain dense in a Hilbert space $\mathfrak{S}$ and its range in a second Hilbert space $\mathfrak{S}^{\prime}$ and let $T^{*}$ be the adjoint of $T$. Let $\alpha^{2}<\lambda<\beta^{2}$ $(0 \leqq \alpha<\beta)$ be an open interval containing at most one nondegenerate eigenvalue of the operator $T^{*} T$ but no other points of its spectrum. If $u$ and $v$ are elements belonging to the domains of $T$ and $T^{*}$, respectively, such that

$$
\|u\| \neq 0, \quad\|v\| \neq 0, \quad(T u, v)=\left(u, T^{*} \imath\right) \geqq 0
$$

and

$$
(\eta-\alpha)(\beta-\eta)>\epsilon^{2}
$$

hold, where

$$
\eta=\frac{(T u, v)}{\|u\| \cdot\|v\|}, \quad \epsilon^{2}=\frac{1}{2}\left[\frac{\|T u\|^{2}}{\|u\|^{2}}+\frac{\left\|T^{*} v\right\|^{2}}{\|v\|^{2}}\right]-\eta^{2},
$$

then there exists certainly an eigenvalue $\lambda^{\prime}$ of the operator $T^{*} T$ in the interval $\left(\alpha^{2}, \beta^{2}\right)$ and the following inequality is valid: ${ }^{6.7}$

where

$$
L \leqq \lambda^{\prime} \leqq U,
$$

$$
L=L(u, v)=\left(\eta-\frac{\epsilon^{2}}{\beta-\eta}\right)^{2}, \quad U=U(u, v)=\left(\eta+\frac{\epsilon^{2}}{\eta-\alpha}\right)^{2}
$$

In order to make this theorem applicable to $(\mathrm{Pr}$.), it is required to construct the spaces $\mathfrak{S}, \mathfrak{S}^{\prime}$ and define the operators $T, T^{*}$ in such a manner that the eigenvalue problem $T^{*} T w=\lambda w$ is equivalent to (Pr.) and the quantities in (5) are practically calculable, at least for the $u$ and $v$ chosen. (Pr.) is nothing but the eigenvalue problem for a self-adjoint operator $H$ (in a real Hilbert space $\mathfrak{S}$ of square integrable functions on $D$ ), defined as

$$
H w=\Delta^{2} w
$$

for $w$ subjected to the same boundary conditions as those of (Pr.), in addition to certain regularity conditions making $\Delta^{2} w$ well-defined. Therefore, our problem is to reduce this preassigned operator $H$ to the form

$$
H=T^{*} T
$$

with a suitable choice of $\mathfrak{S}^{\prime}, T$, and $T^{*}$. Here, $u$ and $v$ may be called "trial functions," or possibly "trial vector functions," in estimating $\lambda^{\prime}$, a better choice of which will yield a better estimate. Finally, $\alpha^{2}$ and $\beta^{2}$ are, loosely speaking, a rough upper bound of the next lower eigenvalue to $\lambda^{\prime}$ and a rough lower bound of the next upper eigenvalue, respectively. Such rough estimates will be obtained by other methods (for instance, the Ritz method for $\alpha^{2}$ ), or occasionally by solving an appropriate auxiliary problem, as in the case for $\beta^{2}$ concerning a square plate, treated later. The decomposition of $H$ into the form $T^{*} T$ is carried out in section 2 for a general shape of $C$. This is followed by some supplementary remarks on theorem

\footnotetext{
${ }^{4}$ Concerning the theory of Hilbert space, we mostly follow the notations and terminology of Stone [4]. For operators between two Hilbert spaces, see Murray [5].

${ }^{5}$ An elementary proof of this theorem will be given elsewhere.

${ }^{6}$ Any eigenvalue of $T^{*} T$ is nonnegative, because $\left(T^{*} T w, w\right)=\|T w\|^{2} \geqq 0$.

${ }^{7} \eta-\frac{\epsilon^{2}}{\beta-\eta}>0$ and $\eta+\frac{\epsilon^{2}}{\eta-\alpha}>0$ follow from (4).
} 
1 for improvement of the approximation. In section 3, confining ourselves to the case of the square plate bounded by $x= \pm 1, y= \pm 1$, we illustrate in detail the method of actual calculation along the lines mentioned above and give a numerical estimate of the smallest positive eigenvalue of (Pr.), taking $\mu=0.225$, with a relative error bound less than $1 / 1000$. Section 4 is devoted to concluding remarks, with a brief reference to other methods with the same object that are applicable to $(\mathrm{Pr}$.).

\section{Decomposition of the Operator and Remarks on Theorem 1}

$F$ The first part of this section is devoted to decomposing $H$, the differential operator of (Pr.) including the boundary conditions, into the form $T^{*} T$ in the sense stated in the introduction, namely, to constructing $T$ and $T^{*}$ so that $H=T^{*} T$, i. e., $T^{*} T w=\lambda w$, implies (Pr.). Strictly speaking, the differential operators appearing here as well as in the sequel must be replaced by their closed extensions with certain extended domains, or in other words, differentiations should be interpreted in a certain generalized sense. Nevertheless, detailed discussions of this situation will not be given, for they seem to be unnecessary for practical application, which is the ultimate object.

2.1. We begin with the following heuristic considerations. ${ }^{8}$ Let $T$ be a closed operator from $\mathfrak{S}$ into $\mathfrak{S}^{\prime}$ and $T^{*}$ its adjoint. Then the eigenvalue problem $T^{*} T w=\lambda w$ is equivalent, at least under a suitable assumption on the spectrum of $T^{*} T$, to the variational problem

$$
\delta\|T u\|^{2}=0, \quad\|u\|^{2}=1,
$$

where $\delta$ is the first variation. Thus, $T^{*} T$ is the "gradient operator" of the quadratic functional $\|T u\|^{2}$ in the sense that $\delta\|T u\|^{2}=2\left(T^{*} T u, \delta u\right)$. On the other hand, as explained in textbooks $[1,8]$, (Pr.) is derived from the variational problem

$$
\delta J[u]=0, \quad \int_{D} u^{2} d x d y=1,
$$

where $J[u]$, which represents (apart from a constant factor) the elastic energy of the plate, is expressed by

$$
J[u]=\int_{D}\left[(\Delta u)^{2}-2(1-\mu)\left\{\frac{\partial^{2} u}{\partial x^{2}} \cdot \frac{\partial^{2} u}{\partial y^{2}}-\left(\frac{\partial^{2} u}{\partial x \partial y}\right)^{2}\right\}\right] d x d y
$$

that is, $H$ is the gradient operator of $J[u]$. Accordingly, $\mathfrak{S}$ being the same as that introduced in defining $H$, it is reasonable to adopt $\mathfrak{S}^{\prime}$ and $T$ such that

$$
\|T u\|^{2}=J[u]
$$

holds. In fact, we proceed as follows. Let $L^{2}(D ; \rho)$ denote the real Hilbert space consisting of functions integrable (in square) on $D$ with norm defined by

$$
\|u\|=\rho \int_{D} u^{2} d x d y
$$

where $\rho$ is a positive constant.

In view of (11) and the fact that $J[u]$ can be written as

$$
J[u]=\int_{D}\left[\mu(\Delta u)^{2}+(1-\mu)\left\{\left(\frac{\partial^{2} u}{\partial x^{2}}\right)^{2}+\left(\frac{\partial^{2} u}{\partial y^{2}}\right)^{2}+2\left(\frac{\partial^{2} u}{\partial x \partial y}\right)^{2}\right\}\right] d x d y,
$$

we define $\mathfrak{S}^{\prime}$ by

$$
\mathfrak{S}^{\prime}=L^{2}(D ; \mu) \times L^{2}(D ; 1-\mu) \times L^{2}(D ; 1-\mu) \times L^{2}(D ; 2-2 \mu),
$$

\footnotetext{
${ }^{3}$ Some general devices useful in such decomposition are proposed in [6].
} 
where $X$ represents the Cartesian product of Hilbert spaces. It should be noticed that an element $v$ of $\mathfrak{S}^{\prime}$ is a four-component vector function $v=\left\{v_{1}, v_{2}, v_{3}, v_{4}\right\}$ defined on $D$. Now the requirement $J[u]=\|T u\|^{2}$ is evidently fulfilled by the operator $T$, defined by

$$
T u=T u(x, y)=\left\{\Delta u, \frac{\partial^{2} u}{\partial x^{2}}, \frac{\partial^{2} u}{\partial y^{2}}, \frac{\partial^{2} u}{\partial x \partial y}\right\} \in \mathfrak{S}^{\prime} .
$$

Elements $u$ belonging to the domain $D$ of $T$ are subjected to certain regularity conditions, but are otherwise arbitrary, i. e., free from any boundary conditions. Though these regularity conditions are too complicated to be specified explicitly in elementary terminology, it will be enough for our purposes to know that the class $\hat{C}$ of functions $u$, such that $u, \partial u / \partial x, \partial u / \partial y$ are continuous and $\partial^{2} u / \partial x^{2}, \partial^{2} u / \partial x \partial y, \partial^{2} u / \partial y^{2}$ are piecewise continuous on $D+C$, is contained in D) namely, $\hat{C}(\mathfrak{D}$.

$T^{*}$ is defined (together with its domain $\mathfrak{D}^{*}\left(\mathfrak{S S}^{\prime}\right)$ by the condition that

$$
(T u, v)=\left(u, T^{*} v\right)
$$

be valid for any $u \in D$. To determine the form of $T^{*}$, we note that

$$
(T u, v)=\int_{D}\left[\mu(\Delta u) v_{1}+(1-\mu) \frac{\partial^{2} u}{\partial x^{2}} v_{2}+(1-\mu) \frac{\partial^{2} u}{\partial y^{2}} v_{3}+2(1-\mu) \frac{\partial^{2} u}{\partial x \partial y} v_{4}\right] d x d y
$$

In reducing the right side of (16) to the form $\int_{D} u \cdot T^{*} v d x d y$, we can resort to devices similar to those usually employed in deriving the differential equation and the boundary conditions of (Pr.) from the variational problem concerning $J[u]$. Before doing this, we introduce some notations, some of which appeared in section 1.

Let

$$
\begin{aligned}
& s=\text { arc length parameter, } \\
& n=n(s)=\left(n_{x}, n_{y}\right)=\text { unit outer normal vector to } C, \\
& t=t(s)=\left(t_{x}, t_{y}\right)=\text { unit tangential vector to } C \text { in the positive direction, }
\end{aligned}
$$

and note that

$$
\frac{\partial}{\partial n}=n_{x} \frac{\partial}{\partial x}+n_{y} \frac{\partial}{\partial y}, \quad \frac{\partial}{\partial t}=t_{x} \frac{\partial}{\partial x}+t_{y} \frac{\partial}{\partial y},
$$

and also

$$
\frac{\partial}{\partial x}=n_{x} \frac{\partial}{\partial n}+t_{x} \frac{\partial}{\partial t}, \quad \frac{\partial}{\partial y}=n_{y} \frac{\partial}{\partial n}+t_{y} \frac{\partial}{\partial t} .
$$

Hereafter it is assumed that the components of $n$ and $t$ are differentiable except at possible angular points $P_{1}, P_{2}, \ldots, P_{n}$ with $s=s_{1}, s_{2}, \ldots, s_{n}$. Also it is known that, for any sufficiently regular functions $F(x, y), G(x, y)$, the identities

$$
\left.\begin{array}{l}
\int_{D} \frac{\partial F}{\partial x} G d x d y=-\int_{D} F \frac{\partial G}{\partial x} d x d y+\int_{C} F \cdot G n_{x} d s, \\
\int_{D} \frac{\partial F}{\partial y} G d x d y=-\int_{D} F \frac{\partial G}{\partial y} d x d y+\int_{C} F \cdot G n_{y} d s,
\end{array}\right\}
$$

are true.

Provided the components of $v$ are sufficiently regular that all procedures used below may be justified, the expression for $(T u, v)$ in $(16)$ becomes, by some small calculations that make use of (18) and (19), 


$$
(T u, v)=\int_{D} u \cdot\left[\mu \Delta v_{1}+(1-\mu)\left\{\frac{\partial^{2} v_{2}}{\partial x^{2}}+\frac{\partial^{2} v_{3}}{\partial y^{2}}+2 \frac{\partial^{2} v_{4}}{\partial x \partial y}\right\}\right] d x d y-\int_{C} u \cdot P d s+\int_{C} \frac{\partial u}{\partial n} Q d s+\int_{C} \frac{\partial u}{\partial t} R d s,
$$

where

$$
\left.\begin{array}{l}
P=P(v, s)=\mu \frac{\partial v_{1}}{\partial n}+(1-\mu)\left\{\frac{\partial v_{2}}{\partial x} n_{x}+\frac{\partial v_{3}}{\partial y} n_{y}+\frac{\partial v_{4}}{\partial x} n_{y}+\frac{\partial v_{4}}{\partial y} n_{x}\right\} \\
Q=Q(v, s)=\mu v_{1}+(1-\mu)\left\{v_{2} n_{x}^{2}+v_{3} n_{y}^{2}+2 v_{4} n_{x} n_{y}\right\}, \\
R=R(v, s)=(1-\mu)\left\{v_{2} n_{x} t_{x}+v_{3} n_{y} t_{y}+v_{4}\left(n_{x} t_{y}+n_{y} t_{x}\right)\right\} .
\end{array}\right\}
$$

Furthermore, because at a point $P$ with $s=\sigma$ we have

$$
\left(\frac{\partial u}{\partial t}\right)_{P}=\left(\frac{d u(s)}{d s}\right)_{s=0}
$$

and consequently

$$
\int_{C} \frac{\partial u}{\partial t} R d s=\int_{C} \frac{d u}{d s} R d s
$$

we can rewrite the last term of (20) by partial integration, taking account of the fact that $C$ is closed and that $R$, as well as the components of $n$ and $t$, is differentiable with respect to $s$ except at $s=s_{1}, \ldots, s_{n}$, so that

$$
\int_{C} \frac{\partial u}{\partial t} R d s=-\int_{C} u \frac{d R}{d s} d s-\sum_{i=1}^{n} u\left(s_{i}\right) D_{i}
$$

where $D_{i}=\lim _{s \rightarrow s_{i}+0} R-\lim _{s \rightarrow s_{i}-0} R, \quad(i=1,2, \ldots, n)$.

The substitution of (22) into (20) results in

$(T u, v)=\int_{D} u\left[\mu \Delta v_{1}+(1-\mu)\left\{\frac{\partial^{2} v_{2}}{\partial x^{2}}+\frac{\partial^{2} v_{3}}{\partial y^{2}}+2 \frac{\partial^{2} v_{4}}{\partial x \partial y}\right\}\right] d x d y-\int_{C} u\left(P+\frac{d R}{d s}\right) d s+\int_{C} \frac{\partial u}{\partial n} Q d s-\sum_{i=1}^{n} u\left(s_{i}\right) D_{i}$.

This expression should be equal to $\left(u, T^{*} v\right)$. Because $u$ and $\partial u / \partial n$ on $C$ are arbitrary, we immediately conclude that this is true if and only if $v$ satisfies the boundary conditions

$$
\left.\begin{array}{rl}
P+\frac{d}{d s} R & =0, \\
Q & =0, \\
D_{i} & =0, \quad(i=1,2, \ldots, n) .
\end{array}\right\}
$$

Then $T^{*}$ is given by

$$
T^{*} v=T^{*}\left\{v_{1}, v_{2}, v_{3}, v_{4}\right\}=\mu \Delta v_{1}+(1-\mu)\left\{\frac{\partial^{2} v_{2}}{\partial x^{2}}+\frac{\partial^{2} v_{3}}{\partial y^{2}}+2 \frac{\partial^{2} v_{4}}{\partial x \partial y}\right\} .
$$

Though an element $v$ belonging to $D^{*}$ must obey certain regularity conditions besides the boundary conditions (24), we will not discuss these in detail. Again it is sufficient to know that any $v$ with components belonging to $\hat{C}$ and satisfying the boundary conditions (24) is contained in $\mathfrak{D} *$.

The construction given above shows that the operators $T$ and $T^{*}$ are certainly the desired ones, but it may be worthwhile to verify directly the equality $H=T^{*} T$, i. e., the equivalence of the eigenvalue problem $T^{*} T w=\lambda w$ to $(\operatorname{Pr}$.). 
Let $w$ belong to the domain of $T^{*} T$. Then $T w$ belongs to $D^{*}$ and consequently is subjected to $(24)$. But

$$
\begin{aligned}
P(T w) & =\mu \frac{\partial \Delta w}{\partial n}+(1-\mu)\left\{\frac{\partial^{3} w}{\partial x^{3}} n_{x}+\frac{\partial^{3} w}{\partial y^{3}} n_{y}+\frac{\partial^{3} w}{\partial x^{2} \partial y} n_{y}+\frac{\partial^{3} w}{\partial x \partial y^{2}} n_{x}\right\} \\
& =\mu \frac{\partial \Delta w}{\partial n}+(1-\mu)\left[\left\{\frac{\partial}{\partial x}\left(\frac{\partial^{2} w}{\partial x^{2}}\right) n_{x}+\frac{\partial}{\partial y}\left(\frac{\partial^{2} w}{\partial x^{2}}\right) n_{y}\right\}+\left\{\frac{\partial}{\partial x}\left(\frac{\partial^{2} w}{\partial y^{2}}\right) n_{x}+\frac{\partial}{\partial y}\left(\frac{\partial^{2} w}{\partial y^{2}}\right) n_{y}\right\}\right] \\
& =\mu \frac{\partial \Delta w}{\partial n}+(1-\mu) \frac{\partial}{\partial n}\left(\frac{\partial^{2} w}{\partial x^{2}}+\frac{\partial^{2} w}{\partial y^{2}}\right) \\
& =\frac{\partial}{\partial n} \Delta w
\end{aligned}
$$

by virtue of (17). Similarly, we obtain

$$
\begin{aligned}
Q(T w) & =\mu \Delta w+(1-\mu)\left\{\frac{\partial^{2} w}{\partial x^{2}} n_{x}^{2}+\frac{\partial^{2} w}{\partial y^{2}} n_{y}^{2}+2 \frac{\partial^{2} w}{\partial x \partial y} n_{x} n_{y}\right\} \\
& =\mu \Delta w+(1-\mu) \frac{\partial^{2} w}{\partial n^{2}}, \\
R(T w) & =(1-\mu)\left\{\frac{\partial^{2} w}{\partial x^{2}} n_{x} t_{x}+\frac{\partial^{2} w}{\partial y^{2}} n_{y} t_{y}+\frac{\partial^{2} w}{\partial x \partial y}\left(n_{x} t_{y}+n_{y} t_{x}\right)\right\} \\
& =(1-\mu) \frac{\partial^{2} w}{\partial t \partial n} .
\end{aligned}
$$

Thus, $w$ satisfies all boundary conditions of $H$ and therefore belongs to the domain of $H$. Conversely, by a similar argument it is seen that any element in the domain of $H$ belongs to the domain of $T^{*} T$. Finally, we observe that

$$
\begin{aligned}
T^{*} T w & =\mu \Delta(\Delta w)+(1-\mu)\left\{\frac{\partial^{2}}{\partial x^{2}}\left(\frac{\partial^{2} w}{\partial x^{2}}\right)+\frac{\partial^{2}}{\partial y^{2}}\left(\frac{\partial^{2} w}{\partial y^{2}}\right)+2 \frac{\partial^{2}}{\partial x \partial y}\left(\frac{\partial^{2} w}{\partial x \partial y}\right)\right\} \\
& =\Delta^{2} w
\end{aligned}
$$

as it should be.

2.2. The following remarks on theorem 1 include some devices that would be of use in obtaining a good estimate, particularly in constructing a desirable pair $u$ and $v$.

REMARK 2.1. The estimates given by theorem 1 are sharp when $\epsilon^{2}$ is small. But it is known [3] that $\epsilon^{2}$ can be written in an alternative form,

$$
\epsilon^{2}=\frac{1}{2}\left(\left\|\frac{1}{\|u\|} T u-\frac{\eta}{\|v\|} v\right\|^{2}+\left\|\frac{1}{\|v\|} T^{*} v-\frac{\eta}{\|u\|} u\right\|^{2}\right),
$$

and $\epsilon^{2}=0$ occurs if, and only if,

$$
T u=\frac{\|u\|}{\|v\|} \eta v \quad \text { and } \quad T^{*} v=\frac{\|v\|}{\|u\|} \eta u \text {. }
$$

This implies that $T^{*} T u=\eta^{2} u$, so that $\eta^{2}$ is equal to an exact eigenvalue $\lambda^{\prime}$ of $T^{*} T$ and $u$ is the corresponding exact eigenfunction $w$. Thus we may expect a good result when $u$ and $v$ approximate, apart from constant factors, $w$ and Tw closely. This interpretation of $u$ and $v$ should be taken into account, in particular when some knowledge concerning the behavior of $w$ is given in some way or other, for instance from physical considerations or experimental results. As for precise discussions of the relations between $u, v$, and $w$, reference is made to the paper by Kato [3]. 
REMARK 2.2. If $u$ and $v$ are not completely preassigned but admit of some free parameters, it would be intrinsically the best way to choose those parameters first to minimize $U$ and then maximize $L$. In practice, however, it appears sufficient as well as convenient for calculation to determine them by minimizing $\epsilon^{2}$. A standard form for such adjustable trial functions is

$$
\left.\begin{array}{l}
u=\alpha_{1} u^{(1)}+\alpha_{2} u^{(2)}+\ldots+\alpha_{m} u^{(m)}, \\
v=\beta_{1} v^{(1)}+\beta_{2} v^{(2)}+\ldots+\beta_{n} v^{(n)},
\end{array}\right\}
$$

where $u^{(1)}, u^{(2)}, \ldots, u^{(m)}$ are fixed functions belonging to $\mathfrak{D}$ and $v^{(1)}, v^{(2)}, \ldots, v^{(n)}$ are fixed vector functions belonging to $D^{*}$, and where $\alpha_{1}, \alpha_{2}, \ldots, \alpha_{m}, \beta_{1}, \beta_{2}, \ldots, \beta_{n}$ are free parameters. If we calculate the quantities in (5) with these $u$ and $v$, then $\|u\|^{2},\|T u\|^{2}$ become quadratic forms in $\left\{\alpha_{i}\right\},\|v\|^{2},\left\|T^{*} v\right\|^{2}$ quadratic forms in $\left\{\beta_{k}\right\}$, and $(T u, v)$ a bilinear form in $\left\{\alpha_{i}\right\}$ and $\left\{\beta_{k}\right\}$. Thus $\epsilon^{2}$ becomes an algebraic expression in those parameters whose minimum is desired. There is little difficulty in the necessary computations, especially when we can resort to an automatic computer, even if $m$ and $n$ in (26) are not very small.

REMARK 2.3. Suppose that a closed subspace $\mathfrak{M}$ of $\mathfrak{S}$ reduces the operator $H=T^{*} T$, that is, let $H w \epsilon \mathfrak{M}$ for any $w$ belonging to the intersection of $\mathfrak{M}$ and the domain of $H$. Then we can regard $H$ as an operator in the Hilbert space $\mathfrak{M}$ denoted by $H_{\mathfrak{M}}$. It is known that eigenvalues and eigenvectors of $H_{\mathfrak{M}}$ are also eigenvalues and eigenvectors of $H$, so that we may speak of an eigenvalue of $H$ in $\mathfrak{M}$. If the eigenvalue $\lambda^{\prime}$ of $H$ in question turns out to be an eigenvalue of $H_{\mathfrak{M}}$, we can derive an estimate for $\lambda^{\prime}$ from theorem 1 more conveniently by restricting $T$ to $\mathfrak{M}$, and $T^{*}$ to a suitable closed subspace $\mathfrak{M}^{*}$ of $\mathfrak{S}^{\prime}$, with the properties

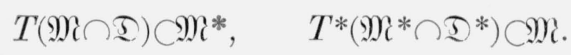

This procedure is particularly advantageous when a degenerate eigenvalue of the $H$ in question is converted into a nondegenerate one of $H_{\mathfrak{M}}$ by a suitable choice of $\mathfrak{M}$ so that application of theorem 1 becomes possible, or when the neighboring eigenvalues of $H_{\mathfrak{M}}$ are more widely separated than those of $H$, thus permitting us to take smaller $\alpha$ and larger $\beta$ so as to make the estimate better. Moreover, though the smallest eigenvalue of $H$ in our problem is equal to zero, the associated eigenfunctions being of the type $a x+b y+c$, we can sometimes find $\mathfrak{M}$ such that the eigenvalue $\lambda^{\prime}$, originally intermediate, becomes the smallest eigenvalue of $H_{\mathfrak{M}}$ and hence its upper bounds can be obtained by

$$
U_{R}=\frac{\|T u\|^{2}}{\|u\|^{2}} \geqq \lambda^{\prime}, \quad(u \in \mathfrak{M} \cap \mathfrak{D}),
$$

the so-called Rayleigh principle. This seems more convenient than the estimation by the right inequality of $(6)$, for it does not involve $v$, an element of $D^{*}$, for which a good choice is not easy because of the complication introduced by the boundary conditions. Therefore, we may prefer $U_{R}$ to $U$ as an upper bound for such an eigenvalue.

Nontrivial $\mathfrak{M}, \mathfrak{M}^{*}$ as mentioned above are often obtained by considering the symmetry properties of the operators $H, T$, and $T^{*}$ reflecting those of $C$, as we shall illustrate in the succeeding section in the case of a square plate.

\section{Vibration of a Square Plate}

In this section the general results obtained so far are applied to the case of a square plate. Let $C$ be the square with vertices $(1,1),(-1,1),(-1,-1)$, and $(1,-1)$, which is the same as that treated by Ritz [2]. Bounds will be calculated for the smallest positive eigenvalue of $H=T^{*} T$ according to theorem 1 and the Remarks in section 2, i. e., lower bounds by means of (6) and upper bounds by means of (6) or (28). 
3.1. We begin with a preliminary consideration. Let

$$
\bar{J}[u]=(1-\mu) \int_{D}\left(u_{x x}^{2}+u_{y y}^{2}\right) d x d y .
$$

Let the self-adjoint operator $\bar{H}$ in $\mathfrak{S}$ be the gradient operator of $\bar{J}[u]$. Explicitly, any element $\bar{w}$ in the domain of $\bar{H}$ is subjected to the boundary conditions

$$
\left.\begin{array}{l}
\frac{\partial^{2} \bar{w}}{\partial x^{2}}=\frac{\partial^{3} \bar{w}}{\partial x^{3}}=0, \quad(x= \pm 1,-1<y<1) \\
\frac{\partial^{2} \bar{w}}{\partial y^{2}}=\frac{\partial^{3} \bar{w}}{\partial y^{3}}=0, \quad(-1<x<1, y= \pm 1),
\end{array}\right\}
$$

and we observe that

$$
\bar{H} \bar{w}=(1-\mu)\left(\frac{\partial^{4} \bar{w}}{\partial x^{4}}+\frac{\partial^{4} \bar{w}}{\partial y^{4}}\right) .
$$

The eigenvalue problem $\bar{H} \bar{w}=\bar{\lambda} \bar{w}$ is reduced, by separation of variables to the eigenvalue problem

$$
\left.\begin{array}{c}
\frac{d^{4} u}{d x^{4}}=k^{4} u, \quad(-<x<1), \\
u^{\prime \prime}( \pm 1)=u^{\prime \prime \prime}( \pm 1)=0,
\end{array}\right\}
$$

and a similar one in the variable $y$. Equation (32) is nothing but the eigenvalue problem treated in the analysis of vibrations of a free bar of length 2, and is completely solved in textbooks $[7,8]$. If we denote the eigenvalues by $k_{n}^{4}(n=0,1,2, \ldots)$ and note that 0 is the unique degenerate one with eigenfunctions $a+b x$, we have

$$
k_{0}=k_{1}=0, \quad 0<k_{2}<k_{3}<k_{4}<\ldots,
$$

where $^{9}$

$$
\begin{array}{ll}
k_{2}=2.3650204, & k_{3}=3.9266023, \\
k_{4}=5.4978039, & k_{5}=7.0685828,
\end{array}
$$

The normalized eigenfunction $u_{n}=u_{n}(x)$ associated with $k_{n}^{4}$ is given by

$$
\left.\begin{array}{l}
u_{0}=\frac{1}{\sqrt{2}}, \quad u_{1}=\sqrt{\frac{3}{2}} x, \\
u_{n}=\frac{\cosh k_{n} \cdot \cos k_{n} x+\cos k_{n} \cdot \cosh k_{n} x}{\sqrt{\cosh ^{2} k_{n}+\cos ^{2} k_{n}}}, \quad \text { for even } n \geqq 2, \\
u_{n}=\frac{\sinh k_{n} \cdot \sin k_{n} x+\sin k_{n} \cdot \sinh k_{n} x}{\sqrt{\sinh ^{2} k_{n}-\sin ^{2} k_{n}}}, \quad \text { for odd } n \geqq 3 .
\end{array}\right\}
$$

It should be noticed that $u_{n}$ is an even or odd function, according as $n$ is even or odd.

The eigenvalues and eigenfunctions of $\bar{H}$ are given by

$$
\bar{\lambda}_{m n}=(1-\mu)\left(k_{m}^{4}+k_{n}^{4}\right), \quad \bar{w}_{m n}=u_{m}(x) u_{n}(y), \quad(m, n=0,1,2, \ldots) .
$$

We note that $\bar{w}_{m n}(m, n=0,1,2 \ldots)$ constitutes a complete orthonormal system of eigenfunctions of the self-adjoint operator $\bar{H}$.

3.2. Comparing two quadratic functionals $J[u]$ and $\bar{J}[u]$, or two operators $H$ and $\bar{H}$, we can not only determine the constant $\beta$ in theorem 1 in a rigorous fashion, but also the sym-

${ }^{\theta} k_{n}$ is obtained as a root of equation $\tan k=\tanh k$ or $\tan k=-\tanh k$, according as $n$ is odd or even. 
metry of the eigenfunction associated with the smallest positive eigenvalue. We note that

$$
\bar{J}[u] \leqq J[u] .
$$

From (36) it follows, by virtue of the properties of $\bar{H}$ stated above, that the spectrum of $H$ consists only of discrete eigenvalues, and also that, if we number all eigenvalues of $H$ and $\bar{H}$ in ascending order, counting any degenerate one repeatedly by its multiplicity, we have

$$
\bar{\Lambda}_{N} \leqq \Lambda_{N},
$$

where $\bar{\Lambda}_{N}$ and $\Lambda_{N}$ are the $N$ th eigenvalue of $\bar{H}$ and the $N$ th eigenvalue of $H$, respectively [7]. Thus the eigenvalues (35) of $\bar{H}$ are the lower bounds of the corresponding eigenvalues of $H$, and hence we can take $\beta$ as

$$
\beta^{2}=\bar{\lambda}_{m n}=(1-\mu)\left(k_{m}^{4}+k_{n}^{4}\right),
$$

if the eigenvalue $\lambda^{\prime}$ of $H$ in question is the $r$ th eigenvalue from below and $\bar{\lambda}_{m n}$ is the $(r+1)$ th eigenvalue of $\bar{H}$.

We turn to the study of the symmetry properties of $H$ and $\bar{H}$, i. e., of closed subspaces specified by the symmetry properties of their elements, and to the reduction of each of these operators. For the square plate under consideration, the boundary conditions of $H$ follow from (2) and the remark there and take the form

$$
\begin{aligned}
\frac{\partial}{\partial x}\left[\frac{\partial^{2} w}{\partial x^{2}}+(2-\mu) \frac{\partial^{2} w}{\partial y^{2}}\right] & =0, & (x= \pm 1,-1<y<1), \\
\frac{\partial}{\partial y}\left[\frac{\partial^{2} w}{\partial y^{2}}+(2-\mu) \frac{\partial^{2} w}{\partial x^{2}}\right] & =0, & (y= \pm 1,-1<x<1), \\
\frac{\partial^{2} w}{\partial x^{2}}+\mu \frac{\partial^{2} w}{\partial y^{2}} & =0, & (x= \pm 1,-1<y<1), \\
\frac{\partial^{2} w}{\partial y^{2}}+\mu \frac{\partial^{2} w}{\partial x^{2}} & =0, & (y= \pm 1,-1<x<1), \\
\frac{\partial^{2} w}{\partial x \partial y} & =0, & (x= \pm 1, y= \pm 1) .
\end{aligned}
$$

In the deduction of the last condition of (39), use is made of the fact that, for instance,

$$
\frac{\partial^{2} w}{\partial n \partial y}=\frac{\partial^{2} w}{\partial x \partial y} \quad \text { on the side } x=1, \quad \frac{\partial^{2} w}{\partial n \partial t}=-\frac{\partial^{2} w}{\partial x \partial y} \quad \text { on the side } y=1,
$$

and hence that the condition

$$
\lim _{\substack{x=1 \\ y \rightarrow 1}} \frac{\partial^{2} n}{\partial n \partial t}=\lim _{\substack{x \rightarrow 1 \\ y=1}} \frac{\partial^{2} w}{\partial n \partial t} \quad \text { means } \quad \frac{\partial^{2} w}{\partial x \partial y}=0 \quad \text { at the vertex }(1,1) .
$$

Owing to the symmetry properties of (39) and those of the differential operator $\Delta^{2}, H$ is reduced by the following subspaces:

$\mathfrak{M}(o, 0)=$ set of functions odd both in $x$ and in $y$,

$\mathfrak{M}(o, e)=$ set of functions odd in $x$ and even in $y$,

$\mathfrak{M}(e, 0)=$ set of functions even in $x$ and odd in $y$,

$\mathfrak{M}(e, e)=$ set of functions even both in $x$ and in $y$, 
which are mutually orthogonal and whose direct sum is the whole space 5 . We see immediately that $\bar{H}$ is also reduced by each of these subspaces, so that the inequality (37) still remains valid, if we regard $H$ and $\bar{H}$ as operators in any of them. Let the eigenvalues of $H$ in these subspaces be denoted as ${ }^{10}$

$$
\begin{array}{ll}
\lambda_{0}<\lambda_{1}<\lambda_{2}<\ldots, & \text { in } \mathfrak{M}(o, o), \\
\mu_{0}<\mu_{1}<\mu_{2}<\ldots, & \text { either in } \mathfrak{M}(o, e) \text { or in } \mathfrak{M}(e, o), \\
\nu_{0}<\nu_{1}<\nu_{2}<\ldots, & \text { in } \mathfrak{M}(e, e)
\end{array}
$$

the union of which is the total spectrum of $H$. On the other hand, the eigenfunction $\bar{w}_{m n}$ of $\bar{H}$ with the eigenvalue $\bar{\lambda}_{m n}$ belongs to $\mathfrak{M}(o, o), \mathfrak{M}(o, e), \mathfrak{M}(e, 0)$, or $\mathfrak{M}(e, e)$, according as $(m=o d d$, $n=$ odd $),(m=$ odd, $n=$ even$)$, ( $m=$ even, $n=$ odd $)$, or ( $m=$ even, $n=$ even $)$.

Because the zero point set $\mathfrak{N}$ of $H$ (the eigenspace with the eigenvalue 0 ) consists of functions of the type $a x+b y+c$, it follows that

and hence that

$$
\left.\begin{array}{l}
\mathfrak{M}(o, o) \perp \mathfrak{N}, \\
\mathfrak{M}(o, e) \cap \mathfrak{N}=\{u ; u=a x\}, \\
\mathfrak{M}(e, o) \cap \mathfrak{N}=\{u ; u=b y\}, \\
\mathfrak{M}(e, e) \cap \mathfrak{N}=\{u ; u=c\},
\end{array}\right\}
$$

$$
\lambda_{0}>0, \quad \mu_{0}=\nu_{0}=0 .
$$

In consequence, the smallest positive eigenvalue of $H$ is equal to $\min \left(\lambda_{0}, \mu_{1}, \nu_{1}\right)$. We can determine the order relations between $\lambda_{0}, \mu_{1}$, and $\nu_{1}$ as follows. Because (40) means that $\mu_{0}$ is degenerate neither in $\mathfrak{M}(o, e)$ nor in $\mathfrak{M}(e, o)$, and $\nu_{0}$ is not degenerate in $\mathfrak{M}(e, e)$, we note, according to (37), that $\bar{\lambda}_{12}\left(=\bar{\lambda}_{21}\right)$ and $\bar{\lambda}_{22}$ are lower bounds of $\mu_{1}$ and $\nu_{1}$, respectively, and hence we have, by means of (33) and (35),

$$
\left.\begin{array}{l}
\mu_{1} \geqq \bar{\lambda}_{12}=\bar{\lambda}_{21}=(1-\mu) k_{2}^{4}=(1-\mu) \times 31.28 \ldots ., \\
\nu_{1} \geqq \lambda_{22}=2(1-\mu) k_{2}^{4}=(1-\mu) \times 62.59 \ldots . .
\end{array}\right\}
$$

On the other hand, the substitution of the trial function $u=x y \in \mathfrak{M}(o, o)$ into (28) yields

$$
\lambda_{0} \leqq(1-\mu) \times 18 .
$$

Combining (41) and (42), we conclude that the smallest positive eigenvalue of $H$ is the smallest eigenvalue $\lambda_{0}$ in $\mathfrak{M}(o, o)$. Moreover, owing to (42) and the relations $\bar{\lambda}_{11}=0, \bar{\lambda}_{13}=\bar{\lambda}_{31}=(1-\mu) \times$ 237.72 . . ., we obtain

$$
\bar{\lambda}_{11}<\lambda_{0}<\bar{\lambda}_{13}=\bar{\lambda}_{31}
$$

which shows that $\bar{\lambda}_{11}$ is the unique eigenvalue of $\bar{H}$ less than $\lambda_{0}$, and hence $\lambda_{0}$ is not degenerate. Also we have, by means of $(37), \bar{\lambda}_{13} \leqq \lambda_{1}$, which enables us to take as $\beta$ in theorem 1 , with respect to $\lambda^{\prime}=\lambda_{0}$, any number such that

$$
\beta^{2} \leqq(1-\mu) \times 237.72 \ldots .
$$

Furthermore, we divide $\mathfrak{M}(o, o)$ into the following two mutually orthogonal subspaces $\mathfrak{M}$ and $\mathfrak{M}_{i}^{\prime}$

$$
\begin{array}{rlrl}
\mathfrak{M} & =\{u ; u \in \mathfrak{M}(o, o), & & u(x, y) \equiv u(y, x)\}, \\
\mathfrak{M}^{\prime} & =\{u ; u \in \mathfrak{M}(o, o), & u(x, y) \equiv-u(y, x)\},
\end{array}
$$

${ }^{10}$ Evidently, the spectra of $H M_{(o, e)}$ and those of $H M_{(e, v)}$ are identical, and any eigenfunction of one of them is converted into an eigenfunction of the other by permutation of $x$ and $y$. 
each of which is seen to reduce both of $H$ and $\bar{H}$.

From (43) it follows that $\lambda_{0}$ is an eigenvalue of $H$ not in $\mathfrak{M}^{\prime}$ but in $\mathfrak{M}$, because the smallest eigenvalue of $\bar{H}$ in $\mathfrak{M}^{\prime}$ is $\bar{\lambda}_{13}$ with eigenfunctions Const. $\left(\bar{w}_{13}-\bar{w}_{31}\right)$, though this is at the same time an eigenvalue of $\bar{H}$ in $\mathfrak{M}$ with eigenfunctions Const. $\left(\bar{w}_{13}+\bar{w}_{31}\right)$. In calculating bounds of $\lambda_{0}$, to which we confine ourselves hereafter, we may therefore restrict $H$ and $T$ to $\mathfrak{M}$.

3.3 We go on to the construction of the trial elements ${ }^{11} u$ and $v$ in theorem 1 . Introducing six elements $u^{(1)}, \ldots, u^{(6)}$ of $\mathfrak{M}$, given by

$$
\begin{array}{ll}
u^{(1)}=P_{1}(x) P_{1}(y), & u^{(2)}=P_{1}(x) P_{3}(y)+P_{3}(x) P_{1}(y), \\
u^{(3)}=P_{3}(x) P_{3}(y), & u^{(4)}=P_{1}(x) P_{5}(y)+P_{5}(x) P_{1}(y), \\
u^{(5)}=P_{3}(x) P_{5}(y)+P_{5}(x) P_{3}(y), & u^{(6)}=P_{5}(x) P_{5}(y),
\end{array}
$$

where $P_{i}$ represents Legendre's polynomial of degree $i(i=1,3,5)$, we put, in accordance with $(26)$,

$$
u=\alpha_{1} u^{(1)}+\alpha_{2} u^{(2)}+\ldots+\alpha_{6} u^{(6)}
$$

with free parameters $\alpha_{1}, \alpha_{2}, \ldots, \alpha_{6}$ to be determined later. This $u$ evidently belongs to $\mathfrak{M} \cap \mathfrak{D}$, because it is a polynomial and surely satisfies the regularity conditions of $\mathfrak{D}$.

The other element $v=\left\{v_{1}, v_{2}, v_{3}, v_{4}\right\}$ is constructed as follows. We can easily verify that the boundary conditions $(24)$ of $D^{*}$ now reduce to the following forms:

$$
\left.\begin{array}{rlrl}
\mu \frac{\partial v_{1}}{\partial x}+(1-\mu) \frac{\partial v_{2}}{\partial x}+2(1-\mu) \frac{\partial v_{4}}{\partial y} & =0, & & (x= \pm 1,-1<y<1), \\
\mu \frac{\partial v_{1}}{\partial y}+(1-\mu) \frac{\partial v_{3}}{\partial y}+2(1-\mu) \frac{\partial v_{4}}{\partial x} & =0, & & (-1<x<1, y= \pm 1), \\
\mu v_{1}+(1-\mu) v_{2} & =0, & & (x= \pm 1,-1<y<1), \\
\mu v_{1}+(1-\mu) v_{3} & =0, & & (-1<x<1, y= \pm 1), \\
v_{4}=0, & & (x= \pm 1, y= \pm 1) .
\end{array}\right\}
$$

In view of the symmetry of $\mathfrak{M}$, on the other hand, we can take as $\mathfrak{M}^{*}$ the subspace composed of elements $v$ with the following properties of symmetry:

$$
\left.\begin{array}{l}
v_{1}, v_{2}, v_{3} \text { are odd both in } x \text { and in } y, \\
v_{4} \text { is even both in } x \text { and in } y, \\
v_{1}(x, y) \equiv v_{1}(y, x), \quad v_{2}(x, y) \equiv v_{3}(y, x), \quad v_{4}(x, y) \equiv v_{4}(y, x) .
\end{array}\right\}
$$

Then the relations (27) obviously hold.

A general element $v$ satisfying the conditions (47), and whose components are polynomials in $x, y$ with the degrees shown below, is expressible as

$$
\left.\begin{array}{l}
v=\left\{v_{1}, v_{2}, v_{3}, v_{4}\right\}, \\
v_{1}=a_{1} x y+a_{2}\left(x y^{3}+x^{3} y\right)+a_{3} x^{3} y^{3}+a_{4}\left(x y^{5}+x^{5} y\right), \\
v_{2}=b_{1} x y+b_{2} x y^{3}+b_{2}^{\prime} x^{3} y+b_{3} x^{3} y^{3}+b_{4} x y^{5}+b_{4}^{\prime} x^{5} y, \\
v_{3}=b_{1} x y+b_{2}^{\prime} x y^{3}+b_{2} x^{3} y+b_{3} x^{3} y^{3}+b_{4}^{\prime} x y^{5}+b_{4} x^{5} y, \\
v_{4}=c_{1}+c_{2}\left(x^{2}+y^{2}\right)+c_{3} x^{2} y^{2}+c_{4}\left(x^{4}+y^{4}\right) .
\end{array}\right\}
$$

\footnotetext{
${ }^{11}$ If we are contented with a rough estimate of $\lambda^{0}$, we can construct the trial elements more easily (see [9]).
} 
Moreover, for such a trial function $v$ the boundary conditions (46) are equivalent to

where $\sigma=\mu /(1-\mu)$.

$$
\left.\begin{array}{rlrl}
\sigma \frac{\partial v_{1}}{\partial x}+\frac{\partial v_{2}}{\partial \imath}+2 \frac{\partial v_{4}}{\partial y} & =0, & & (x=1,-1<y<1), \\
\sigma v_{1}+v_{2} & =0, & & (x=1,-1<y<1), \\
v_{4} & =0, & & (x=y=1),
\end{array}\right\}
$$

Substituting (48) into (49), putting $x=1$ and equating the coefficients of $y, y^{3}, y^{5}$ to zero or putting $x=y=1$, we obtain the conditions for $v$ given by (48) to satisfy (49) and hence to belong to D*. The results are the following six equalities: ${ }^{12}$

$$
\begin{aligned}
& \sigma\left(a_{1}+3 a_{2}+5 a_{4}\right)+\left(b_{1}+3 b_{2}^{\prime}+5 b_{4}^{\prime}\right)+\left(4 c_{2}+4 c_{3}\right)=0, \\
& \sigma\left(a_{1}+a_{2}+a_{4}\right)+\left(b_{1}+b_{2}^{\prime}+b_{4}^{\prime}\right) \quad=0, \\
& \sigma\left(a_{2}+3 a_{3}\right)+\left(b_{2}+3 b_{3}\right)+8 c_{4}=0, \\
& \sigma\left(a_{2}+a_{3}\right)+\left(b_{2}+b_{3}\right) \quad=0, \\
& \sigma a_{4}+b_{4} \quad=0, \\
& c_{1}+2 c_{2}+c_{3}+2 c_{4}=0 \text {. }
\end{aligned}
$$

By inspection, we find the following 8 independent families, (1), (2), . . , (8), of parameters satisfying the system (50) of 6 equations in 14 unknowns:

$$
\begin{array}{lll}
\text { (1) } a_{1}=1, & b_{1}=-\sigma, \\
\text { (2) } a_{2}=1, & b_{2}=b_{2}^{\prime}=-\sigma, & \\
\text { (3) } & b_{1}=-1, b_{2}^{\prime}=1, & c_{1}=1, c_{2}=-1 / 2, \\
\text { (4) } a_{3}=1, & b_{3}=-\sigma & \\
\text { (5) } & & c_{1}=c_{3}=1, c_{2}=-1, \\
\text { (6) } a_{4}=1, & b_{4}=b_{4}^{\prime}=-\sigma, & \\
\text { (7) } & b_{1}=-1, b_{4}^{\prime}=1, & c_{1}=1, c_{3}=-1, \\
\text { (8) } & b_{2}=-2, b_{3}=2, & c_{1}=1, c_{4}=-1 / 2 .
\end{array}
$$

The absent members of each family are equal to 0 .

In this way, we have the following 8 elements $v^{(k)}=\left\{v_{1}^{(k)}, v_{2}^{(k)}, v_{3}^{(k)}, v_{4}^{(k)}\right\}(k=1,2, \ldots, 8)$ contained in $\mathfrak{M}^{*} \cap \mathfrak{D} *$.

$$
\begin{aligned}
& v^{(1)}=\{x y, \quad-\sigma x y, \quad-\sigma x y, \quad 0\}, \\
& v^{(2)}=\left\{x y^{3}+x^{3} y, \quad-\sigma\left(x y^{3}+x^{3} y\right), \quad-\sigma\left(x y^{3}+x^{3} y\right), \quad 0\right\}, \\
& v^{(3)}=\left\{0, \quad-x y+x^{3} y, \quad-x y+x y^{3}, \quad 1-\left(x^{2}+y^{2}\right) / 2\right\}, \\
& v^{(4)}=\left\{x^{3} y^{3}, \quad-\sigma x^{3} y^{3}, \quad-\sigma x^{3} y^{3}, \quad 0\right\}, \\
& v^{(5)}=\left\{0, \quad 0, \quad 0, \quad\left(1-x^{2}\right)\left(1-y^{2}\right)\right\}, \\
& v^{(6)}=\left\{x y^{5}+x^{5} y, \quad-\sigma\left(x y^{5}+x^{5} y\right), \quad-\sigma\left(x y^{5}+x^{5} y\right), \quad 0\right\}, \\
& v^{(7)}=\left\{0, \quad-x y+x^{5} y, \quad-x y+x y^{5}, \quad 1-x^{2} y^{2}\right\}, \\
& v^{(8)}=\left\{0, \quad 2\left(-x y^{3}+x^{i} y^{3}\right), \quad 2\left(-x^{3} y+x^{3} y^{3}\right), \quad 1-\left(x^{4}+y^{4}\right) / 2\right\} .
\end{aligned}
$$

12 The result of equating to zero the coefficients of $y^{5}$ in the first and second conditions of (49) coincides and gives the fifth condition of (50). 
With these 8 elements as basis, we construct the trial function $v$ in accordance with (26):

$$
v=\beta_{1} v^{(1)}+\beta_{2} v^{(2)}+\ldots .+\beta_{8} v^{(8)}
$$

with free parameters $\beta_{1}, \beta_{2}, \ldots, \beta_{8}$.

Making use of the trial functions $u$ and $v$ given by (45) and (51) respectively, we get, after the necessary integrations,

$$
\begin{aligned}
\|T u\|^{2} & =\sum_{i, k=1}^{6} A_{i k} \alpha_{i} \alpha_{k}=(1-\mu) \sum A_{i k}^{\prime} \alpha_{i} \alpha_{k}, \quad\left(A_{i k}=A_{k i}\right), \\
\|u\|^{2} & =\sum_{i, k=1}^{6} B_{i k} \alpha_{i} \alpha_{k}, \quad\left(B_{i k}=B_{k i}\right), \\
T^{*} v \|^{2} & =\sum_{i, k=1}^{8} C_{i k} \beta_{i} \beta_{k}=(1-\mu) \sum C_{i k}^{\prime} \beta_{i} \beta_{k}, \quad\left(C_{i k}=C_{k i}\right), \\
\|v\|^{2} & =\sum_{i, k=1}^{8} D_{i k} \beta_{i} \beta_{k}=(1-\mu) \sum D_{i k}^{\prime} \beta_{i} \beta_{k}, \quad\left(D_{i k}=D_{k i}\right), \\
(T u, u) & =\left(u, T^{*} v\right)=\sum_{i=1}^{6} \sum_{k=1}^{8} E_{i k} \alpha_{i} \beta_{k}=(1-\mu) \sum \sum E_{i k}^{\prime} \alpha_{i} \beta_{k}
\end{aligned}
$$

\begin{tabular}{|c|c|c|c|c|c|c|}
\hline & 1 & 2 & 3 & 4 & 5 & 6 \\
\hline 1 & 8 & 16 & 8 & 16 & 16 & 8 \\
\hline 2 & & $400 \sigma+312$ & 96 & $1120 \sigma+672$ & 192 & 96 \\
\hline 3 & & & $\frac{600}{7} \sigma+\frac{2616}{7}$ & $360 \sigma+96$ & $240 \sigma+816$ & 288 \\
\hline 4 & & & & $4648 \sigma+3336$ & $1008 \sigma+336$ & 240 \\
\hline 5 & & & & & $\frac{22248}{11} \sigma+\frac{37296}{11}$ & $\frac{1680}{11} \sigma+\frac{17520}{11}$ \\
\hline 6 & & & & & & $840 \sigma+2640$ \\
\hline
\end{tabular}

with $A_{i k}^{\prime}, B_{i k}, C_{i k}^{\prime}, D_{i k}^{\prime}, E_{i k}^{\prime}$ given by the tables.

$A_{i k}$

$B_{i k}$

\begin{tabular}{|c|c|c|c|c|c|c|c|}
\hline$i$ & 1 & 2 & 3 & 4 & 5 & 6 & \\
\cline { 1 - 2 }$B_{i i}$ & $\frac{4}{9}$ & $\frac{8}{21}$ & $\frac{4}{49}$ & $\frac{8}{33}$ & $\frac{8}{77}$ & $\frac{4}{121}$ & $B_{i k}=0 \quad(i \neq k)$ \\
\hline
\end{tabular}


$C_{i k}^{\prime}$

\begin{tabular}{|c|c|c|c|c|c|c|c|c|c|c|c|}
\hline$i$ & 3 & 3 & 3 & 3 & 5 & 5 & 5 & 7 & 7 & 8 & \multicolumn{1}{c}{$\begin{array}{c}\text { For other values } \\
\text { of } i \text { and } k, \\
C_{i k}^{\prime}=0 .\end{array}$} \\
\hline$k$ & 3 & 5 & 7 & 8 & 5 & 7 & 8 & 7 & 8 & 8 \\
\hline$C_{i k}^{\prime}$ & 64 & $\frac{178}{3}$ & $\frac{256}{3}$ & $\frac{348}{5}$ & $\frac{256}{9}$ & $\frac{512}{9}$ & $\frac{256}{5}$ & $\frac{8704}{63}$ & $\frac{4096}{35}$ & $\frac{17664}{175}$ & \\
\hline
\end{tabular}

$D_{i k}^{\prime}$

\begin{tabular}{|c|c|c|c|c|c|c|c|c|}
\hline$i$ & 1 & 2 & 3 & 4 & 5 & 6 & 7 & 8 \\
\hline 1 & $\frac{4}{9} \tau$ & $\frac{8}{15} \tau$ & $\frac{16}{45} \sigma$ & $\frac{4}{25} \tau$ & 0 & $\frac{8}{21} \tau$ & $\frac{32}{6} \overline{3}^{\sigma}$ & $\frac{32}{75} \sigma$ \\
\hline 2 & & $\frac{368}{525} \tau$ & $\frac{64}{175} \sigma$ & $\frac{8}{35} \tau$ & 0 & $\frac{496}{945} \tau$ & $\frac{512}{945} \sigma$ & $\frac{256}{525}^{\sigma}$ \\
\hline 3 & & & $\frac{144}{35}$ & $\frac{16}{175}$ & $\frac{128}{45}$ & $\frac{32}{135} \sigma$ & $\frac{992}{189}$ & $\frac{2528}{525}$ \\
\hline 4 & & & & $\frac{4}{49^{\tau}}$ & 0 & $\frac{8}{45^{\tau}}$ & $\frac{32}{225} \sigma$ & $\frac{32}{245} \sigma$ \\
\hline 5 & & & & & $\frac{512}{225}$ & 0 & $\frac{256}{75}$ & $\frac{1024}{315}$ \\
\hline 6 & & & & & & $\frac{656}{1617^{\tau}}$ & $\frac{192}{53 y} \sigma$ & $\frac{64}{189} \sigma$ \\
\hline 7 & & & & & & & $\frac{119744}{17325}$ & $\frac{1088}{175}$ \\
\hline 8 & & & & & & & & $\frac{63424}{11025}$ \\
\hline
\end{tabular}

$E_{i k}^{\prime}$

\begin{tabular}{|c|c|c|c|c|c|c|c|}
\hline$i$ & 1 & 1 & 1 & 1 & 2 & 2 \\
\cline { 1 - 2 } & 3 & 5 & 7 & 8 & 7 & 8 & $\begin{array}{c}\text { For other values of } i \text { and } k, \\
E_{i k}^{\prime}=0 .\end{array}$ \\
\hline$E_{i k}^{\prime}$ & $\frac{16}{3}$ & $\frac{32}{9}$ & $\frac{64}{9}$ & $\frac{32}{5}$ & $\frac{64}{21}$ & $\frac{64}{35}$ & \\
\hline & & & &
\end{tabular}

Here $\sigma$ and $\tau$ are constants given by $\sigma=\frac{\mu}{1-\mu}, \tau=\frac{1+\mu}{(1-\mu)^{2}}$.

In the numerical computation we take $\mu=0.225$, the value used by Ritz. Then the quantity $2 \epsilon^{2}$ becomes an algebraic expression of the form

$$
2 \epsilon^{2}=\frac{x^{\prime} A x}{x^{\prime} B x}+\frac{y^{\prime} C y}{y^{\prime} D y}-2 \frac{\left(x^{\prime} E y\right)^{2}}{\left(x^{\prime} B x\right)\left(y^{\prime} D y\right)},
$$


where $x, y$ are the column vectors with components $\left\{\alpha_{i}\right\},\left\{\beta_{k}\right\}$, respectively, $x^{\prime}, y^{\prime}$ are their transposed vectors, and $A, B, C, D, E$ are matrices with numerical elements.

In order to minimize $\epsilon^{2}$, we choose the following procedure. First we minimize the first term on the right-hand side of (52). This gives rise to an eigenvalue problem of order 6 , for which we determine the lowest eigenvalue $m$ (which is the minimum value in question) and the associated eigenvector $x=x_{1}$. Then we put $x=x_{1}$ into (52); this converts (52) into a fractional form of which the numerator and denominator are known quadratic forms in $y$. We minimize this expression; this is equivalent to solving another eigenvalue problem of order 8 , and we determine the lowest eigenvalue $m^{\prime}$ and the associated eigenvector $y=y_{1}$. In the next step we should put $y=y_{1}$ into (52), converting (52) into a ratio of two quadratic forms in $x$, and determine its minimum $m^{\prime \prime}$. This procedure could be repeated indefinitely, and the sequence $m^{\prime}, m^{\prime \prime}, \ldots$ is certainly nonincreasing. It is not clear whether these values converge to the true minimum of $2 \epsilon^{2}$. But this is not important, for actually we need not obtain this true minimum; it is sufficient for our purpose that a very small value of $2 \epsilon^{2}$ is given by some set of $x$ and $y$.

The actual computation was done by using SEAC, and it turned out that the value of $m^{\prime}$ was sufficiently small, so that further computation was not necessary. To avoid the uncontrollable error arising from the use of the machine, the components of $x_{1}, y_{1}$ thus obtained have been rounded off to five significant figures, and the value of $2 \epsilon^{2}$ for these arguments has been calculated anew on a desk calculator. The final values are

$$
\begin{array}{lll}
\alpha_{1}=3.6100000, & \beta_{1}=2.0362000, \\
\alpha_{2}=-0.2071000, & \beta_{2}=-1.1853000, \\
\alpha_{3}=-0.0357000, & \beta_{3}=-1.6269000, \\
\alpha_{4}=0.0335000, & \beta_{4}=0.3121000, \\
\alpha_{5}=-0.0012000, & \beta_{5}=0.5907000, \\
\alpha_{6}=-0.0017000, & \beta_{6}=0.0112000, \\
& \beta_{7}=0.0176000, \\
& \beta_{8}=0.1233000, \\
\epsilon^{2}=0.009172, & \eta=3.6266247 .
\end{array}
$$

We can take $\alpha=0$ and $\beta=13.5$ in (7) for $U$ and $L$ in conformity with (44). Thus we obtain

$$
U=12.455433, \quad L=12.430613,
$$

where the rounding-off errors are within $5 \times 10^{-5}$ by the most conservative estimates. Thus

$$
12.4305<\lambda_{0}<12.4555 \text {. }
$$

For the upper bound we can also use $U_{R}$, given by (28). This gives

$$
U_{R}=12.454620,
$$

which is somewhat sharper than $U$. In this way we arrive at the final result:

$$
12.4306<\lambda_{0}<12.4547 \text {. }
$$

If we adopt $\lambda_{0}=12.4427$, the average of upper and lower bounds, as the smallest positive eigenvalue of $H=T^{*} T$, the relative error is less than $1 / 1000$.

The corresponding value of the fundamental frequency of a thin elastic square plate $(\mu=0.225)$ with free edges is

$$
\nu=\frac{\sqrt{\lambda_{0}}}{2 \sqrt{3} \pi \sqrt{1-\mu^{2}}} \sqrt{\frac{E}{\rho}} \frac{h}{a^{2}}=0.3420 \times \sqrt{\frac{E}{\rho}} \frac{h}{a^{2}},
$$

with relative error less than $1 / 2000$. Here $2 a=$ edge length, $2 h=$ thickness, $\rho=$ density, and $E=$ Young's modulus. 
A remark is necessary regarding the relation between the result of Ritz [2] and that of this study. Ritz gives the estimate $\lambda_{0} \leqq 12.43$, and this appeared exceedingly sharp in view of the results shown here (53). Ritz used products of eigenfunctions of free bars as trial functions $u^{(i)}$. This suggested that these trial functions might lead to a sharper upper bound than the one presented in this paper. Because the last figure in Ritz' result is unreliable, the same calculation was carried out for purposes of comparison by retaining more significant figures. The result obtained gives only $\lambda_{0}<12.488$, a less sharp estimate than the one herein. This seems to show that, as far as the fundamental frequency of a square plate is concerned, use of polynomial trial functions is preferable to products of eigenfunctions of free bars.

\section{Concluding Remarks}

4.1. This method is also applicable to a plate whose edge is not wholly free but subjected to conditions of a more general type. For instance, let us assume that $C$, the boundary of the plate under consideration, consists of three arcs, $C_{0}, C_{1}$, and $C_{2}$, and that the edge is free, supported, and clamped along $C_{0}, C_{1}$, and $C_{2}$, respectively. Among all angular points on $C$, we denote those on $C_{0}$ by $P_{1}, P_{2}, \ldots, P_{n}$ with $s=s_{1}, s_{2}, \ldots, s_{n}$. Then as in the case of the free plate discussed above, the vibration of this plate is characterized by the eigenvalue problem of a self-adjoint operator $\hat{H}$ in $\mathfrak{S}$. Again, $\hat{H}$ is the gradient operator of a quadratic functional $\hat{J}$ $[u]$, which is the restriction of $J[u]$ to the class of functions $u$ satisfying the boundary conditions

$$
\left.\begin{array}{cc}
u=0 & \text { on } C_{1}+C_{2}, \\
\frac{\partial u}{\partial n}=0 & \text { on } C_{2} .
\end{array}\right\}
$$

Thus, following a course parallel to that of section 2 , we can reduce $\hat{H}$ to $\hat{T}^{*} \hat{T}$ and make theorem 1 applicable. The results of this decomposition are as follows. $\mathfrak{5}$ and $\mathfrak{S}^{\prime}$ being the same as before, $\hat{T} u$ and $\hat{T}^{*} v$ are formally identical with $T u$ and $T^{*} v$, respectively, and now any $u$ belonging to the domain of $\hat{T}$ is subjected to the boundary conditions (54) and any $v$ belonging to the domain of $\hat{T}^{*}$ is subjected to the boundary conditions

$$
\left.\begin{array}{rl}
Q=0 & \text { on } C_{0},+C_{1}, \\
P+\frac{d}{d s} R=0 & \text { on } C_{0}, \\
D_{i}=0, \quad(i=1,2, \ldots, n),
\end{array}\right\}
$$

where $P, Q, R, D_{i}$ are those defined in section 2 .

Applications of theorem 1 to the cases of such mixed boundary conditions will be treated elsewhere.

In addition, if both $C_{0}$ and $C_{1}$ are empty, i. e., the whole edge is clamped, another decomposition seemingly more convenient is possible. Namely, by means of (19) and the conditions $u=\partial u / \partial n=0$ on $C$, we have

$$
\int_{D} \frac{\partial^{2} u}{\partial x^{2}} \cdot \frac{\partial^{2} u}{\partial y^{2}} d x d y=\int_{D}\left(\frac{\partial^{2} u}{\partial x \partial y}\right)^{2} d x d y=\int_{D} u \frac{\partial^{4} u}{\partial x^{2} \partial y^{2}} d x d y
$$

and hence, in consideration of (10), we obtain

$$
\hat{J}[u]=\int_{D}(\Delta u)^{2} d x d v^{\prime}
$$


Thus, taking $\mathfrak{S}=\mathfrak{S g}^{\prime}=L^{2}(D ; 1)$ we can decompose $\hat{H}$ into the desired form by using $T_{c}$, and $T_{c}^{*}$ such that

$$
\begin{aligned}
& T_{c} u=\Delta u, \quad\left(u=\frac{\partial u}{\partial n}=0 \quad \text { on } C\right), \\
& T_{c}^{*} v=\Delta v .
\end{aligned}
$$

4.2. Before his derivation of theorem 1, one of the authors gave also the ffollowing theorem 2 [10], which stands in a close connection with the former.

Theorem 2. Let $H$ be a self-adjoint operator of a Hilbert Space 5 , and let $(\alpha, \beta)$ be an open interval containing at most a nondegenerate eigenvalue of $H$ but no other point of its spectrum. Let $w$ be any vector of the domain $\mathfrak{D}_{H}$ of $H$ with $\|w\|=1$, and set $\eta=(H w, w), \epsilon^{2}=\|(H-\eta) w\|^{2}=$ $\|H w\|^{2}-\eta^{2}$. If $\epsilon^{2}<(\eta-\alpha)(\beta-\eta)$, then there is certainly an eigenvalue $\lambda^{\prime}$ of $H$ in $(\alpha, \beta)$ that satisfies the inequalities

$$
\eta-\frac{\epsilon^{2}}{\beta-\eta} \leqq \lambda^{\prime} \leqq \eta+\frac{\epsilon^{2}}{\eta-\alpha} .
$$

This theorem can be interpreted as follows. If we can construct a "trial function" $w$ in accordance with the conditions stated above, we would obtain an estimate of the eigenvalue $\lambda^{\prime}$ in question by means of (56). Comparing theorem 1 with theorem 2 , we note that, if a good choice of $w$ is easy for the operator $H$ under consideration, theorem 2 may be preferable inasmuch as it does not require the decomposition $H=T^{*} T$, and also it involves fewer quantities to be calculated. However, this is not the case so far as (Pr.) is concerned, for the difficulties in obtaining a suitable $w$ seem greater than those in obtaining a suitable pair of $u$ and $v$ (essentially $v$ ) because of the increased complication of the boundary conditions of $\mathfrak{D}_{H}$. Furthermore, we have four components $v_{1}, v_{2}, v_{3}, v_{4}$ at our disposal in constructing the trial vector function $v$ belonging to $\mathfrak{D}^{*}$, whereas we must make a single function $w$ satisfy all boundary conditions in order that $w \in \mathfrak{D}_{H}$. For instance, let us consider the case of the square plate dealt with in section 3 and look for a polynomial trial function $w$ contained in $\mathfrak{D}_{H} \cap \mathfrak{M}$. For any $w$ belonging to $\mathfrak{M}$, the boundary conditions (39) of $\mathfrak{D}_{H}$ reduce to

$$
\left.\begin{array}{rl}
\frac{\partial^{3} w}{\partial x^{3}}+(2-\mu) \frac{\partial^{3} w}{\partial x \partial y^{2}}=0, & (x=1,-1<y<1), \\
\frac{\partial^{2} w}{\partial x^{2}}+\mu \frac{\partial^{2} w}{\partial y^{2}}=0, & (x=1,-1<y<1), \\
\frac{\partial^{2} w}{\partial x \partial y}=0, & (x=y=1) .
\end{array}\right\}
$$

On the other hand, a general form of a polynomial of degree $2 N-2$, belonging to $\mathfrak{M}$ is given by

$$
w=\sum_{r=2}^{N} \sum_{\substack{m, n=1 \\ m \leqq n}}^{m+n=r} A_{m n}\left(x^{2 m-1} y^{2 n-1}+x^{2 n-1} y^{2 m-1}\right) .
$$

Substituting (58) into (57), putting $x=1$ and equating the coefficients of all powers of $y$ to zero, or putting $x=y=1$, we derive the conditions for $w$ given by (58) to satisfy (57). This gives rise to a system of $2 N-3$ equations in $N^{2} / 4$ unknowns, or a system of $2 N-4$ equations in $\left(N^{2}-1\right) / 4$ unknowns, according as $N$ is even or odd. The smallest value of $N$ permitting nontrivial solutions is seen to be 7 . Therefore, in order to obtain a function of the type (58) in $\mathfrak{D}_{H} \cap \mathfrak{M}$, we have to solve at least a system of 10 equations in 12 unknowns, leaving two homogeneous parameters, i. e., essentially only 1 free parameter in a 12-degree polynomial.

4.3. Weinstein and Aronszajn have developed an approximating method [11], ${ }^{14}$ with a wide range of application, which also enables us to calculate upper and lower bounds of eigen-

14 See [11] for a standard list of references concerning this method. 
values of a certain kind of operators. Although the arguments used in establishing the convergence of their method are theoretically interesting, it appears that, from the viewpoint of practical applications, the essential parts of their method are the procedures leading from rough bounds to those that improve as sharply as possible. In this sense their method seems to have some similarity to the present one, and hence it will be worthwhile to reveal the mutual relations between these two methods and to compare their merits. However, we reserve this for some future occasion and remark here only that, with respect to lower bounds of eigenvalues in the case of the square plate treated in section 3, the eigenvalue problem of the operator $\bar{H}$ can play the role of the "auxiliary problem" in their method, of which the complete set of the exact eigenvalues and eigenvectors is required to be known.

\section{References}

[1] K. O. Friedrichs, Die Randwert und Eigenwert Probleme aus der Theorie der Elastischen Platten (Anwend ung der Direkten Methoden der Variationsrechnung), Math. Ann. 98, 206 to 247 (1927).

[2] W. Ritz, Theorie der Transversalschwingungen einer quadratischen Platte mit freien Rändern, Ann. Phys. 28, 737 to 786 (1909).

[3] T. Kato, On some approximate methods concerning the operators $T * T$, Math. Ann. 126, 253 to 262 (1953).

[4] M. H. Stone, Linear transformations in Hilbert space (New York, 1932).

[5] F. J. Murray, Linear transformations between Hilbert spaces and the application of this theory to linear partial differential equations, Trans. Am. Math. Soc. 37, 301 to 338 (1935).

[6] H. Fujita, Contribution to the theory of upper and lower bounds in boundary value problems, J. Phys. Soc. Japan 10, 1 to 8 (1955).

[7] R. Courant and D. Hilbert, Methods of mathematical physics, I, chap. V and VI, 1st English ed. (Interscience Publishers, Inc., New York, N. Y., 1953).

[8] Lord Rayleigh, Theory of sound, I (Dover Publications, New York, N. Y., 1954).

[9] Y. Nakata and H. Fujita, On upper and lower bounds of the eigenvalues of a free plate, J. Phys. Soc. Japan 10, 823 to 824 (1955).

[10] T. Kato, On the upper and lower bounds of eigenvalues, J. Phys. Soc. Japan 4, 334 to 339 (1949).

[11] N. Aronszajn, Approximation methods for eigenvalues of completely continuous symmetric operators, Proceedings of the symposium on spectral theory and differential problems, 179 to 202 (Math. Dept. Oklahoma Agri. and Mech. College, 1955).

Washington, October 3, 1956. 